Archives

40 | 2007

Dépendance(s)

\title{
Un nouveau regard porté par l'institution militaire du XVIII ${ }^{\mathrm{e}}$ siècle sur ses soldats
}

\section{Naoko Seriu}

\section{(2) OpenEdition Journals}

Édition électronique

URL : http://journals.openedition.org/ccrh/3403

DOl : $10.4000 /$ ccrh.3403

ISSN : $1760-7906$

Éditeur

Centre de recherches historiques - EHESS

\section{Édition imprimée}

Date de publication : 25 avril 2007

Pagination : 187-195

ISSN : 0990-9141

Référence électronique

Naoko Seriu, "Un nouveau regard porté par l'institution militaire du XVIII ${ }^{\mathrm{e}}$ siècle sur ses soldats », Les Cahiers du Centre de Recherches Historiques [En ligne], 40 | 2007, mis en ligne le 13 octobre 2011, consulté le 20 avril 2019. URL : http://journals.openedition.org/ccrh/3403 ; DOI : 10.4000/ccrh.3403

Ce document a été généré automatiquement le 20 avril 2019

Article L.111-1 du Code de la propriété intellectuelle. 


\title{
Un nouveau regard porté par l'institution militaire du XVIII ${ }^{\mathrm{e}}$ siècle sur ses soldats
}

\author{
Naoko Seriu
}

1 Dans quelle mesure la notion de « dépendance » peut-elle enrichir notre connaissance du passé ? Quelques pistes de réflexion se dégagent à partir de recherches qui portent sur les expériences des soldats au XVIII' en France ${ }^{1}$. Mais au préalable, il convient de s'arrêter sur le terme de « dépendance » qui est - comme le rappelle la présentation de la journée - « chargée par les valeurs qu'elle a prises » au cours de l'histoire.Si épuiser cette notion est impossible, expliciter une équivoque liée à ce mot peut être dès lors une première étape de l'analyse.

\section{Autour du mot « dépendance »}

2 Entendu comme antinomie de "l'indépendance » ou de "l'autonomie », le terme de « dépendance » est connoté négativement. Cet état apparait comme à l'opposé de l'idéal défendu par le libéralisme, à savoir une société fondée sur la maximalisation de la liberté des individus, poursuivant rationnellement leur bien-être sans interférer avec les autres². De façon évidente, on constate que « l'indépendance » constitue une valeur morale qu'on peut s'approprier de multiples façons, qu'il s'agisse de justifier un parcours d'enrichissement individuel - à l'image du mythe du « self-made-man » - ou, dans un tout autre contexte, à l'occasion d'une lutte collective, de légitimer un mouvement de contestation. De la lutte féministe à la lutte des pays colonisés, le thème de « l'indépendance » ou de "l'émancipation » justifie la volonté de rompre la domination et de réclamer des droits qui avaient été jusque-là refusés. Disposer de sa liberté, agir en conséquence, constituent autant d'arguments en faveur de l'action politique.

3 La hiérarchie de valeurs qui se construit à travers de multiples usages n'est donc pas en faveur de «la dépendance ». Celle-ci en arrive à désigner un ordre régressif servile, et parfois fonctionne comme un stigmate purement négatif. Ce mot si usité et usé, au lieu de 
décrire une situation, véhicule alors tout simplement une condamnation morale. Décréter que telle ou telle personne est dépendante comme pour essentialiser cet état, cela s'apparente à un acte de pouvoir dont les chercheurs doivent bien évidemment s'abstenir. Il est d'ailleurs très intéressant de rassembler les situations où on utilise ce mot pour désigner, et par-là même, implicitement condamner l'autre 3 .

Pour illustrer ces propos, on peut citer une phrase, en apparence anodine, de Jürgen Habermas : dans la préface à une réédition, de 1990, de L'Espace public, où il va réagir aux discussions antérieurement suscitées par son ouvrage, se trouve un passage qui répondait aux féministes, lesquelles lui reprochaient d'avoir négligé les personnes exclues de la sphère publique et notamment d'avoir sous-estimé la présence des femmes. Donnant raison à ces critiques et prenant une attitude de compromis, il écrivait :

[...] la littérature féministe a aiguisé notre perception du caractère patriarcal de la sphère publique elle-même - d'une sphère publique qui a émergé de ce public de lecteurs, que les femmes ont contribué à forger, en le dépassant aussitôt pour prendre en charge des fonctions politiques. On peut ainsi se poser la question de savoir si les femmes furent exclues de la sphère publique bourgeoise de la même façon que les ouvriers, les paysans et le "bas peuple», donc les " hommes dépendants $»^{4}$.

5 Si la centralité des propos réside dans le débat féministe, cette dernière expression - « bas peuple», donc les "hommes dépendants»- suscite de nombreuses interrogations. Qu'est-ce que cette équation ? Qu'est-ce qu'un homme dépendant ? Ou plutôt qu'est-ce que cet acte de qualification unilatérale ? Pour le philosophe, la catégorie du « bas-peuple " ne contient-elle donc pas les femmes ? Y a-t-il d'un côté les "hommes dépendants » dépouillés de toute compétence, et de l'autre «les femmes ", qui à l'image de celles qui tiennent les salons, seraient beaucoup moins dépendantes et dotées de capacité intellectuelle? Dans tous les cas, pour le philosophe, cette question ne se pose pas aux élites masculines qui s'affirment d'emblée comme des sujets autonomes, comme des «personnes rationnelles, libres et égales entre elles » pour reprendre une expression de John Rawls, penseur libéral contemporain, commentant Kant ${ }^{5}$. Si cette idée s'accorde aux propos d'Habermas, elle correspond également à une vision consensuelle de l'ordre social hiérarchisé et sexué.

6 Pour se prémunir de ce qui se rapproche d'un clichét, citons l'article « indépendance » de l'Encyclopédie :

La pierre philosophale de l'orgueil humain ; la chimère après laquelle l'amour propre court en aveugle; le terme que les hommes se proposent toujours, \& qui empêche leurs entreprises \& leurs désirs d'en avoir jamais, c'est l'indépendance.

Cette perfection est sans doute bien digne des efforts que nous faisons pour l'atteindre, puisqu'elle renferme nécessairement toutes les autres ; mais par-là même elle ne peut point se rencontrer dans l'homme essentiellement limité par sa propre existence. Il n'est qu'un seul être indépendant dans la nature ; c'est son auteur. Le reste est une chaîne dont les anneaux se lient mutuellement, \& dépendent les uns des autres, excepté le premier, qui est dans la main même du créateur? ${ }^{7}$.

7 L'article fait bien apparaître l'importance de " l'indépendance " comme valeur identitaire. Qu'il s'agisse d'un " terme que les hommes se proposent toujours ", peut impliquer qu'ils rejettent son pendant, la « dépendance » envers les autres. Ce piège est toujours présent, dans une époque où « l'individualité » est devenue une valeur morale sacrée. 
8 Expliciter le malaise lié à cette expression conduit, d'une part, à se garder de tout jugement moral qui risque de réduire la complexité d'une vie marquée par la souffrance et la lutte à quelque chose de l'ordre de l'immaturité et de l'incompétence. Par ailleurs, le constat de cette équivoque oblige à être précis quant au sens que l'on donne à ce mot.

\section{La vie militaire au XVIII ${ }^{\mathrm{e}}$ siècle}

Pour mener une réflexion dans le cadre des études sur l'armée française au XVIII' siècle, on peut partir d'une définition simple. L'article « dépendre » dans Le petit Robert donne par exemple les trois définitions suivantes :

-

-

- dépendre de : ne pouvoir se réaliser sans l'action ou

l'intervention d'une personne ou d'une chose ;

- faire partie (de qqch) au sens où « ce parc dépend de la propriété »;

- être sous l'autorité, la domination, l'emprise.

Comment peut-on, en recourant à ces différentes acceptions, appréhender les relations qui structurent l'armée au XVIII siècle, les relations hiérarchiques, en particulier ? Y a-t-il des phénomènes qu'on ne voyait pas auparavant mais qui, à travers ce prisme, deviennent visibles? À la lumière de cette question, il s'agit, en un premier lieu, d'appréhender ce que signifiait la vie militaire pour les soldats, et ensuite, de présenter comment les officiers pouvaient considérer leur relations avec les hommes de troupe dans la deuxième moitié du XVIII siècle.

\section{Les soldats : un statut de dépendance?}

11 Concernant, tout d'abord, la majorité de ceux qui constituent l'armée, donc les soldats, examinons s'il est pertinent d'aborder leur situation en terme de "dépendance ». À se référer précisément à la troisième acception, on peut dire que les soldats dépendent des officiers et qu'ils sont sous leur autorité et domination. En témoigne le simple fait que les soldats doivent obéir aux ordres des officiers. L'ordonnance du roi de 1727 sur « les crimes \& délits militaires » stipule :

Tous soldats, cavaliers \& dragons seront tenus, sous peine de la vie, d'obéir aux officiers des régiments \& compagnies dont ils seront, en tout ce qui leur sera par eux ordonné pour le service de Sa Majesté, soit dans les armées, en route, dans les quartiers \& dans les garnisons ${ }^{8}$.

Leur autorité sur les soldats se confirme encore au niveau des pratiques punitives relevant de la discipline du corps. À la différence des «crimes et délits militaires » qui font l'objet de procès devant un conseil de guerre, la punition des «simples fautes de service \& de discipline " se règle sur le simple rapport d'un officier au commandant. L'acte de punir s'inscrit alors dans l'ordre hiérarchique militaire, allant du haut vers le bas. D'après l'ordonnance du $1^{\text {er }}$ mars 1768 concernant «le service dans les places", " tous les officiers d'un grade supérieur, pourront punir ceux d'un grade inférieur ${ }^{9}:$ ces normes ne sont pas lettre morte. L'analyse d'environ 900 interrogatoires de déserteurs issus des archives de la maréchaussée, notamment de Bretagne, permet de constater la banalité de la violence qui s'exerce sur le corps des soldats : ils doivent craindre non seulement les punitions - prison, salle de discipline, coups de plat de sabre - mais aussi les mauvais traitements physiques et verbaux des officiers pendant le service ${ }^{10}$. 
13 Prendre acte de cette réalité de la violence ${ }^{11}$ exercée à l'encontre d'un individu vulnérable conduit à mettre en cause la pertinence de l'expression "dépendance ». Le terme risquerait d'euphémiser les faits de sévices qu'il faudrait aborder en terme de rapports de violence.

14 Par ailleurs, les témoignages des soldats font surgir l'importance du corps qui apparaît comme la cible principale sur laquelle s'exercent diverses formes du pouvoir de l'institution. L'exercice a pour but de redresser la posture du soldat ${ }^{12}$. À travers l'appel qui représente des contraintes certes mineures mais répétitives - trois ou cinq fois par jour la place du corps est assignée, rattachée à un lieu fixé par l'institution ${ }^{13}$. Lors des multiples inspections, encore, le corps s'expose au regard des officiers, lesquels l'évaluent et peuvent le punir pour « la moindre chose » comme des taches sur les vêtements ou des traces de rouille sur l'arme ${ }^{14}$. L'extrême attention portée aux choses se confirme aussi dans le fait que la perte des effets peut être punie sévèrement ${ }^{15}$. L'habit qui couvre son corps n'appartient pas au soldat, qui doit veiller à sa conservation.

Ce dernier point, sur lequel on ne peut pas trop insister, mérite d'autant plus d'attention qu'il est assez courant de souligner les avantages que présenterait la vie militaire pour une population démunie, parmi lesquels l'uniforme peut être énuméré. De là, il n'y a qu'un pas pour en déduire que le soldat est dépendant de l'institution au sens non pas de la domination mais au sens où il ne peut pas vivre, - s'habiller, se nourrir - sans elle . Le risque éventuel est alors que la dépendance fasse office de catégorie fourre-tout qui émietterait l'objet de l'analyse.

$16 \mathrm{Au}$ travers des mots de soldat, une autre question se pose pourtant : de quoi le soldat dispose-t-il ? Il n'est pas libre de ses gestes, lorsqu'il est puni parce qu'il a mal posé la main pendant l'exercice. Il n'est pas libre de parole, lorsqu'il est puni pour une simple observation faite à la hiérarchie. Il n'a pas la liberté de se déplacer, lorsqu'il est puni pour « un quart d'heure » de retard à l'appel. Il ne dispose pas de ses habits ni de ses effets. S’il faut trouver un terme pour rendre compte de ces faits, repérables non pas seulement dans les textes de règlements, mais aussi dans les pratiques, ce qu'il faut évoquer ce n'est pas le terme de « dépendance » mais celui de " dépossession »- ou plutôt de rapports de "possession" et de "dépossession ». D’une manière absolue, le fait qu'il lui faille accepter sa propre mort est une ultime forme de dépossession. Mais c'est une parenthèse qu'il faut refermer puisqu'il s'agit de réfléchir sur la dépendance.

17 Revenons à la première définition du verbe dépendre, au sens où chacun est lié à l'autre. Si cela relève de la condition de tout homme qui s'inscrit dans la relation avec autrui, les soldats nouent également des liens à la fois à l'intérieur et à l'extérieur de l'institution. D'une part, il est très important de se faire reconnaître par les autres soldats. Bien des témoignages révèlent les interactions perpétuelles qui vont du plaisir d'être ensemble jouer, raconter des histoires dans la chambrée, boire - jusqu'à l'agressivité du rituel du duel qui permet notamment à la recrue de se faire une place au sein de la compagnie ${ }^{16}$. L'importance du regard des autres se confirme également dans la souffrance mêlée de culpabilité des soldats rejetés par les autres et désertant pour cela ${ }^{17}$. En outre, pour les soldats, il y a les proches - parents, femme, enfants - qui forment des liens que l'engagement et l'éloignement ne sauraient effacer ${ }^{18}$. Dans les mots de ceux qui désertent par souci pour ces personnes intimes, apparaît cette « existence humaine interrompant et dépassant son effort d'être » pour reprendre l'expression d'Emmanuel Lévinas ${ }^{19}$. Son éthique fondée sur la vulnérabilité de l'homme va au rebours de la logique libérale de l'individu, qui, lui, est cantonné dans son « effort d'être ». La dépendance apparaît alors 
sous un jour nouveau, qui fait qu'elle éclaire les divers modes de relation que chacun invente pour vivre ensemble et pour y trouver un sens.

\section{Jeux de dépendance des officiers face aux soldats}

18 Si l'on quitte, à présent, le point de vue des soldats, pour s'interroger sur la façon dont les officiers considèrent leur relation avec eux, on peut rappeler la première définition du mot "dépendre", à savoir «ne pas pouvoir se réaliser sans [...] l'intervention d'une personne [...]». Dans un contexte de crise, après la défaite de la guerre de Sept Ans, nombreux sont les officiers qui mènent une réflexion sur la nécessaire réforme de l'institution : résoudre le problème de la désertion, perfectionner l'exercice, instaurer une discipline stricte sont autant de questions discutées par les élites militaires qui envoient des mémoires au ministre. Dans ce discours, - même s'il ne s'agit pas d'une opinion majoritaire - la dépendance des officiers vis-à-vis des soldats apparaît comme un constat tout à fait novateur à partir duquel les relations hiérarchiques doivent être reconfigurées.

Dans un mémoire anonymeintitulé Discipline daté de 1776, l'auteur recommande aux officiers de bien traiter les soldats :

[...]aimez les, soyez leur attaché, n'oubliez jamais que ce sont des hommes que c'est eux qui contribuent avec plaisir, quoi qu'avec des fatigues, des peines et des mal êtres incroyables à la gloire dont vous officiers retirez le principal et le plus grand avantage, non seulement, le soldat jouit de la moindre partie des récompenses, tandis qu'il éprouve le plus grand mal être pendant qu'il se porte bien, il n'éprouve que peu et point de soins étant malade et blessé. Quel est l'homme qui passe sur un champ de bataille, dont le cœur n'ait senti et partagé les douleurs aiguës que les blessés expriment par leurs cris et prières à obtenir la mort de la main du passant, qui n'ait déploré l'humanité en voyant ces malheureux souvent deux ou trois fois vingt-quatre heures sans secours et entassés sur des chariots où ils meurent 1000 fois pour une par les douleurs que leur causent le transport et l'espèce des secours qu'ils reçoivent après? Que de bras que de jambes coupés pour épargner les soins et les frais d'une longue guérison? Que de braves gens envoyés dans l'autre monde par ces bourreaux de Médecins et Chirurgiens ${ }^{20}$.

La recommandation de bien traiter les soldats exprimée ici découle du fait que ces derniers sont indispensables pour que les officiers réalisent leur gloire. Ce constat de dépendance vis-à-vis des soldats constitue un changement de regard majeur : dans la mesure où les soldats éprouvent plus de peine que les officiers, qui sont les principaux bénéficiaires, on ne saurait mieux insister sur l'importance de bien traiter les hommes de troupes. Dès lors, « aimer les soldats », utiliser les compliments, récompenser, sont autant de moyens pour conforter la discipline.

21 Lucides sur leur propre situation de dépendance, les élites militaires le sont également quant à l'utilisation de ce lien. Et il est des officiers qui soulignent la nécessité de créer un état de dépendance qui attacherait les soldats à l'institution. Cette fois, ce sont les soldats qui sont désignés comme sujets de dépendance. Mais il faut souligner que cet état est voulu par l'autorité. Comment celle-ci peut-elle alors procéder pour faire dépendre les soldats de l'institution? La récompense est la réponse qu'elle apporte et expérimentera, faisant ainsi écho aux propositions de certains officiers.

Dans un mémoire intitulé Moyens d'empêcher la désertion daté de 1761, de la Balme, lieutenant de la maréchaussée, écrit :

Le Français est naturellement affectionné à son Roy, fait pour la guerre, capable d'affronter les plus grands dangers et de supporter les plus grandes fatigues, 
pourvu que les dangers et les fatigues lui présentent une perspective honorable. Né libre et fait pour l'estre par sa grande légèreté, tout ce qui a l'air de servitude le rebute et le décourage : mais sçait on le prendre par le point d'honneur, il en devient alors esclave sans qu'il paroisse s'en douter ${ }^{21}$. mettant en place la vétérance est une application de cette idée de récompense ${ }^{23}$. Dorénavant, les soldats qui ont accompli trois engagements seront décorés d'une plaque et investis du droit de porter l'épée pour le reste de leur vie. Par ailleurs, la haute paye, liée au nombre d'engagements, est instaurée pour les soldats en service : ceux qui, au terme de huit ans de service, contractent un nouvel engagement de la même durée, recevront un sou par jour de haute-paye, en plus de leur solde. Pour ceux qui entament leur troisième terme, la haute-paye est portée à deux sous par jour. Les vétérans qui quittaient le service conservaient alors leur solde ainsi augmentée. Si ces récompenses matérielles s'évanouissent par la suite puisque Saint-Germain les supprime en 1776, on voit bien cette volonté politique qui fait que l'institution est abordée comme un lieu où toute la vie de l'individu est sensée se dérouler. Ainsi que le souligne un officier dans un discours adressé aux soldats et mettant en valeur ces bénéfices créés en 1771: «on se prépare un avenir heureux en servant avec fidélité $»^{24}$. différente. Un mémoire du chevalier de Buffevent, capitaine du régiment de Lorraine, daté du 14 février 1772, relève ces origines obscures des récompenses militaires. Tout en se félicitant de l'ordonnance du 16 avril 1771, il écrit au ministre :

D'après les grâces particulières qui viennent d'être accordées aux soldats [...]selon leur gradation de service, l'on peut donc maintenant apercevoir par un calcul relatif à leurs propres intérêts, que du nombre de ceux qui jouissent de la première récompense due à leur ancienneté, il n'en seroit plus déserté, sans devoir du moins être exposé à des regrets qui augmenteroient toujours à proportion du tems où ils se rapprocheroient de la vétérance qui fait pour eux le comble du bonheur ${ }^{25}$.

La notion arbitraire de l'intérêt est ici capitale : la législation vise à créer un état où les hommes trouvent « leur propre intérêt » à persévérer dans l'armée. L'intérêt, quand bien 
même personnel, n'est point une donnée, un désir qu'il suffirait de supposer présent en chacun. C'est l'institution qui le fabrique et l'organise dans le but précis d'attacher les hommes au service : il s'agit précisément de faire dépendre l'individu de l'institution à travers la création de petits bénéfices. La « dépendance » est alors une forme de relation que la monarchie souhaite expérimenter auprès des soldats qu'elle ne saurait domestiquer par la force.

\section{Conclusion}

En prenant comme fil conducteur la question de la dépendance, nous avons parcouru quelques-uns des aspects de la vie militaire. S'il convient d'éviter le préjugé du libéralisme, il s'agit d'une problématique importante qui permet d'éclairer un pan méconnu des relations humaines. La polysémie du terme qui en fait la richesse exige en même temps d'en délimiter le sens, et de choisir son champ d'application. Aussi, fallait-il indiquer quelques impasses qui empêcheraient de saisir les rapports de dépendance comme un processus dynamique. Si, par exemple, le terme de dépendance ne semble pas être propice pour rendre compte de la réalité du corps des soldats, il l'est en revanche pour décrire la densité des relations qui les entourent.

Par ailleurs, une perspective d'analyse, prometteuse, s'esquisse concernant le regard que porte l'officier sur ses subordonnés. Dans la seconde moitié du XVIII siècle, l'officier, prenant acte du fait qu'il dépend du soldat, cherche paradoxalement à créer un état de dépendance pour le soldat lui-même. Souligner cette double dimension conduit à interroger les dispositifs expérimentés à la fin de l'Ancien Régime. Suivre la dialectique par là amorcée est d'autant plus difficile qu'il s'agit d'une expérience éphémère. Tout en laissant ouverte la question de la réception, isoler la stratégie du pouvoir soucieux d'obtenir l'obéissance volontaire de la part des soldats est nécessaire. Ainsi, une réflexion à propos de la dépendance questionne obligatoirement les formes d'identité se construisant face à la réaction du pouvoir.

\section{NOTES}

1. Naoko Seriu, «Faire un soldat. Une histoire des hommes à l'épreuve de l'institution militaire ( XVIII ${ }^{\mathrm{S}}$ ) », Thèse de l'École des hautes études en sciences sociales, 2005.

2. International Encyclopedia of the social and behavioral sciences, Oxford, Pergamon, 2001, t.13, «Liberalism », p. 8797.

3. Patrick Declerck, dans un contexte actuel, signale que l'hébergement de longue durée est refusé aux sans-abris sous le prétexte de favoriser l'« autonomie du sujet » (Les naufragés. Avec les clochards de Paris, Plon, Paris, 2001, p. 30).

4. Jürgen Habermas, L'espace public : archéologie de la publicité comme dimension constitutive de la société bourgeoise, Paris, Payot, 1993, préface à l'édition de 1990, p. VIII. 
5. John Rawls, Théorie de la justice, Le Seuil, 1987, p. 288-289. Critiquant la position de Rawls, Michael Sandel écrit: "L'indépendance tant célébrée du sujet déontologique est une illusion libérale » (Le Libéralisme et les limites de la justice, Paris, Le Seuil, 1998, p. 36).

6. Allant à l'encontre de ce mouvement majeur, Emmanuel Lévinas présumera pourtant que les hommes sont dépendants l'un envers l'autre, cherchant une toute autre éthique fondée sur la vulnérabilité et le dénuement de l'homme.

7. Diderot et d'Alembert, Encyclopédie ou dictionnaire raisonné des sciences des arts et des métiers par une société de gens de lettres, Paris, 1751-1780, 34 vol., t. VIII, p. 671.

8. Claude de Briquet, Code militaire, nouvelle édition augmentée, Paris, chez Durand, 1761, 7 vol., t. III, p. 112-124.

9. Service Historique de la Défense (SHD), X2 23 : Ordonnance du 1er mars 1768, Tit. 21, art. 29.

10. Voir Naoko Seriu, op.cit., p. 174-226.

11. À propos de l'armée piémontaise au XVIII siècle, Sabina Loriga écrit : "Les soldats ne connaissent que rarement la violence de la punition » (Soldats. Un laboratoire disciplinaire : l'armée piémontaise au XVIII'siècle., Paris, Mentha, 1991, p. 149). Cette appréciation ne semble pas décrire la condition des soldats en France. D'après les interrogatoires des soldats arrêtés comme déserteurs, la banalité, l'injustice et la violence des punitions relevant de «simples fautes de service \& de discipline » sont une dimension quotidienne de la vie militaire.

12. Naoko Seriu, op.cit., p. 92-98 et p. 202-207.

13. Ibid., p. 207-216.

14. Ibid., p. 183-202.

15. Ibid., p. 216-222.

16. Naoko Seriu, op.cit., p. 269-276.

17. Ibid., p. 276-295.

18. Ibid., p. 306-376.

19. Emmanuel Lévinas, Entre nous, Paris, Grasset, 1991, p. 10.

20. SHD, 1M 1786 : Discipline, 1776, p. 65 sq.

21. SHD, $1 \mathrm{M} 1783$ : de la Balme, Moyens d'empêcher la désertion ou du moins de la réduire à très peu de chose, 1761, p. 4.

22. Sabina Loriga, op. cit., p. 23 (supra n. 11). Concernant les officiers de l'armée française, Hervé Drévillon a récemment apporté un éclairage précieux, L'impôt du sang, Paris, Taillandier, 2006.

23. Sur la mise en œuvre du système de récompenses, voir Jean-Pierre Bois, Les anciens soldats dans la société française au XVIII siècle, Paris, Economica, 1990.

24. SHD 1M 1786 : Baron d'Aigalliers, Discours au soldat français, p. 17.

25. SHD 1M 1786 : Chevalier de Buffevent, Réflexions sur la discipline, 1772, p. 2.

\section{AUTEUR}

\section{NAOKO SERIU}

Université Paris-III/CRH 